\title{
Monitoring: a fundamental functionality for orchestrating services on Fog Computing
}

\author{
Breno Costa, Aletéia P. F. Araújo \\ ${ }^{1}$ Departamento de Ciencia da Computação - Universidade de Brasília (UnB) \\ Brasília - DF - BraSil \\ brenogscosta@gmail.com, aleteia@unb.br
}

\begin{abstract}
Fog Computing is a distributed paradigm that provides computational resources in the users' vicinity. To provide services to the users, take place the orchestration of fog resources and services, a set of functionalities that coordinate the dynamic infrastructure and take actions to guarantee the SLAs agreed. Monitoring is an orchestration functionality of prime importance. It is the basis for resource management actions, collecting resources status and providing the data needed to an adequate management. This work proposes a monitoring architecture for fog computing environments that addresses the needs for an adequate fog resource and service orchestration.
\end{abstract}

\section{Introduction}

Fog Computing is a new computational paradigm that complements cloud computing, providing computational resources on the network edge, closer to the users. As a distributed infrastructure, Fog Computing has to deal with heterogeneity of network links and processing capacity of its composing nodes [Bonomi et al. 2012].

These characteristics bring complexity to fog management and it is addressed by the orchestration of services and resources. Orchestration is a management function, composed of several complementary functionalities and responsible for dealing with infrastructure dynamicity, for taking timely actions and for assuring that Service Level Agreements (SLAs) are respected [Velasquez et al. 2017].

There are several proposals of fog service orchestration in the literature, although the majority of them are only conceptual. The vast majority of them point out monitoring as a relevant component, but they frequently suppose that a fog monitoring solution will be available, without offering insights and implementation.

Monitoring is an orchestration functionality of primer importance. It collects updated status information about fog nodes and communication links and send them to the orchestrator. With an updated view of fog infrastructure and service execution, the orchestrator can take proper actions to guarantee the SLAs, e.g. offloading a service to a richer resource node and optimizing service placement according to historic data about node failures.

Besides the heterogeneity of nodes being monitored, there are other related monitoring concerns about frequency, topology and method of communication. There is a trade-off between the frequency of information updates and the overhead to the nodes and to the orchestrator related to generating, transmitting and processing the status updates. Will the nodes send the updates in a stated frequency or the orchestrator will ask 
for them only when it can deal with this burden? Monitoring topology must adhere to the orchestrator topology?

There are in the literature only a few proposals to address fog computing monitoring. As far as we know, none of them has approached the monitoring needs of a fog orchestrator: a monitoring system that offers varied forms of communication, with negligible interference in latency, exchanged data, so that the orchestrator has adequate options according to the scenario of the dynamic environment. This work proposes a fog computing monitoring architecture that supports fog resource and service orchestration, filling the gaps found in the literature. The main contributions are:

- A Fog Computing monitoring taxonomy, describing the main characteristics, purposes and use cases of this functionality;

- An open source implementation of the proposed architecture;

- An evaluation, on a real testbed, of the main monitoring concerns in the context of fog orchestration.

\section{Theoretical Foundation}

\subsection{Fog Computing}

Fog Computing is a distributed computing paradigm that provides resources for computing, storage and connectivity at the network edge. It can be considered an extension of cloud computing towards the users' locality.

It provides computing resources for applications that cannot perform properly with the high latency provided by cloud-only environments. It resides in between the cloud and users and the cloud will do long-term storage and non-latency-dependent processing [Naha et al. 2018].

The layered (or hierarchical) representation of fog computing is the most widely used approach [Naha et al. 2018]. In this context, a three-tiered architecture is the common representation of fog computing environment [Mahmud and Buyya 2016]: IoT Layer, Fog Layer and Cloud Layer.

\subsubsection{Essential Characteristics}

Regarding the characteristics of Fog Computing, although some publications are divergent [Bonomi et al. 2012], [Mukherjee et al. 2018] and [Hu et al. 2017], the ones most used by academics are those indicated by the NIST [Iorga et al. 2018], described bellow:

- Low Latency: as fog computing devices are often located at the edge of the network, the analysis and response to data generated by these devices is much faster than from a cloud service;

- Geographic Distribution: in contrast to cloud computing, services and applications driven by fog computing require widely geo-distributed deployments;

- Heterogeneity: supports the collection and processing of data originating from different sources and acquired through various types of network communication resources;

- Interoperability and Federation: components must be able to interoperate and services must be federated between domains; 
- Real-Time Interactions: fog computing applications involve real-time interactions, rather than batch processing;

- Scalability: supports scalability of computational resources, changes in workloads and variations in network and device conditions.

\subsection{Orchestration in Fog Computing}

Fog orchestration is a management function responsible for service life cycle. To provide requested services to the user and assure the SLAs, it must monitor the underlying infrastructure, react timely to its changes and comply with privacy and security rules. It is composed of several functionalities, e.g. resource management, monitoring, service life cycle management [Velasquez et al. 2017, Wen et al. 2017], as can be seen on Figure 1, a generic architecture created from the analysis of 50 orchestration works available on the literature.

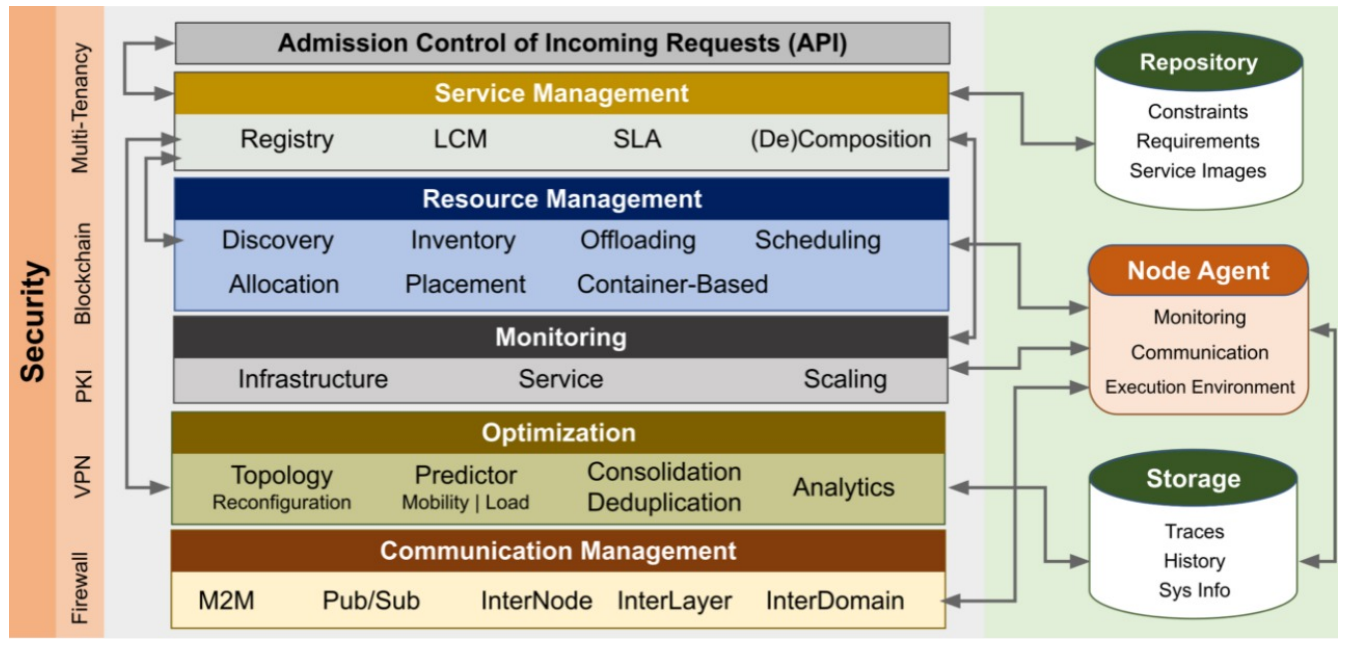

Figure 1. A generic Fog Computing orchestration architecture.

\subsection{Monitoring Fog Computing infrastructures}

Infrastructure monitoring is the basis to support several goals: make an efficient use of resources, measure resource and service performance, generate accurate bills [Syed et al. 2017] and implement fault tolerance processes.

Monitoring is a functionality of prime importance in fog orchestration. It is responsible for collecting infrastructure metrics, e.g. cpu usage, latency etc, and to maintain updated the status of resource and service availability. This information can be useful for decision making about service placement, service offloading, scalability issues, SLA and QoS management, other fog computing orchestration functionalities seen on Figure 1. Thus, monitoring can be seen as a glue that bounds all of them together.

To properly deal with fog characteristics, e.g. resource-restricted and heterogeneous devices, variety and instability of connections, fog monitoring solutions must attend to specific requirements that are not available on cloud monitoring solutions in place [Abderrahim et al. 2017], thus requesting specific approaches. According to Abderrahim et al., the solutions for monitoring Fog infrastructures must have the following properties:

- Scalability - to deal with the increased number of fog nodes; 
- Resilience to server apparitions/removals - mobility is a potential characteristic of a fog node;

- Resilience to network changes/failures - since this is a fog characteristic;

- Modularity - there must be room for adaptation or parameterization as there is resource heterogeneity;

- Locality - The monitoring service must be as nearest as possible to the resources and services being monitored.

\section{Preliminary Analysis}

Syed et. al. analyzed cloud monitoring solutions and created a thematic taxonomy, showed on Figure 2. Confronting this taxonomy to Fog Computing characteristics (Section 2.1.1) and relevant fog monitoring proposals [Brandón et al. 2018, Souza et al. 2018, Forti et al. 2021], one can see that the themes Monitoring Perspectives and Types of Cloud do not apply to the Fog context, since the lack of a business model is one of the challenges of Fog Computing [Lewis 2019]. New themes are needed to make the taxonomy proper to use on Fog Computing, as for instance the inclusion of Monitored Metrics to identify and separate the set of monitored metrics for infrastructure monitoring and for service monitoring. Inclusion of new categories inside the themes proposed is also necessary, as the inclusion of Fault Tolerance to the theme Monitoring Purposes and the inclusion of Hybrid category on Monitoring Architecture.

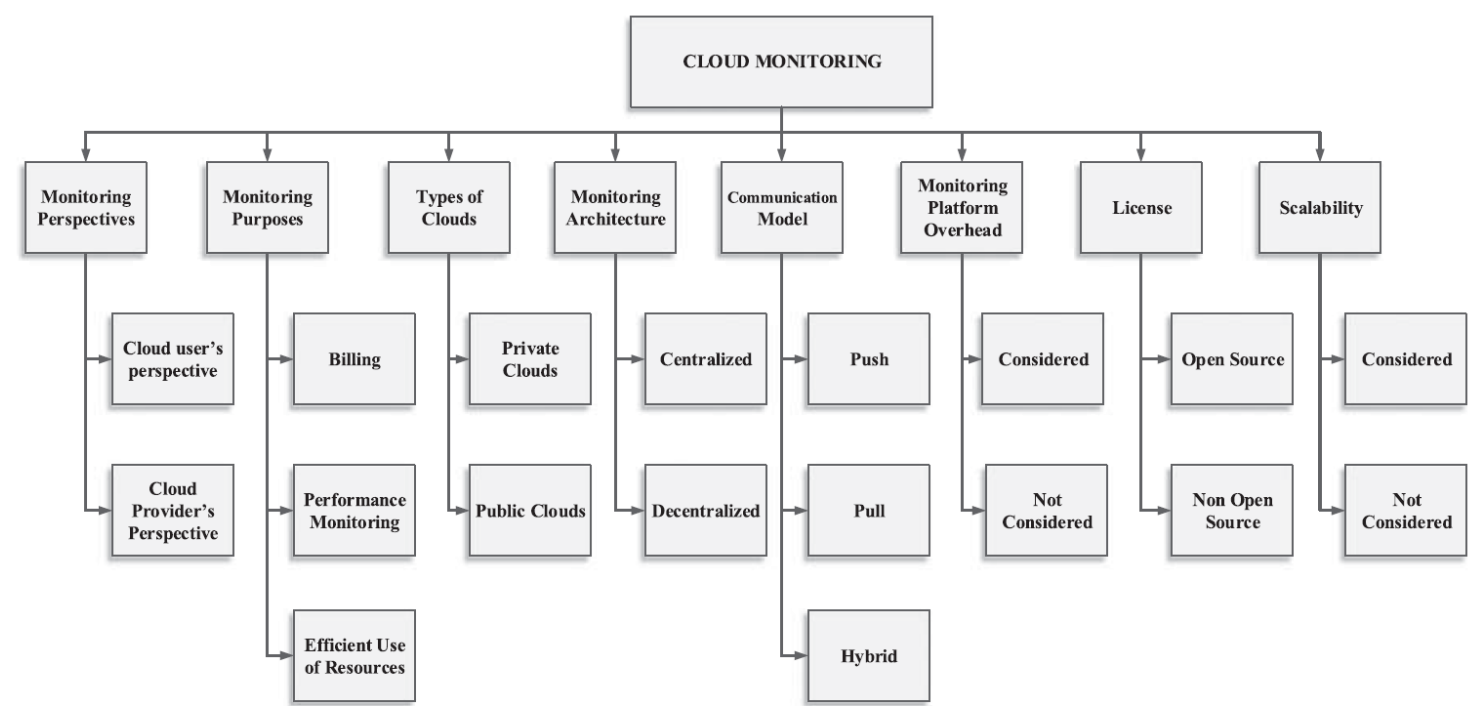

Figure 2. Thematic taxonomy of a cloud monitoring solution [Syed et al. 2017].

\section{Final Considerations}

This work will analyze fog monitoring proposals and create an appropriate and specific taxonomy describing the characteristics, the purposes and the use cases of fog computing monitoring solutions.

The taxonomy will help in the definition of a monitoring architecture capable of support several fog computing monitoring needs, offering a broader and comprehensive conceptual solution to approach the theme. 
Finally, an use case of monitoring on the context of fog computing orchestration will be implemented as an open source and evaluated on an actual testbed, providing results to validate the viability and the added value of such a solution.

\section{References}

Abderrahim, M., Ouzzif, M., Guillouard, K., Francois, J., and Lebre, A. (2017). A holistic monitoring service for fog/edge infrastructures: a foresight study. In 2017 IEEE 5th International Conference on Future Internet of Things and Cloud (FiCloud), pages 337-344. IEEE.

Bonomi, F., Milito, R., Zhu, J., and Addepalli, S. (2012). Fog computing and its role in the internet of things. In Proceedings of the First Edition of the MCC Workshop on Mobile Cloud Computing, MCC '12, pages 13-16, New York, NY, USA. ACM.

Brandón, Á., Pérez, M. S., Montes, J., and Sanchez, A. (2018). Fmone: A flexible monitoring solution at the edge. Wireless Communications and Mobile Computing, 2018.

Forti, S., Gaglianese, M., and Brogi, A. (2021). Lightweight self-organising distributed monitoring of fog infrastructures. Future Generation Computer Systems, 114:605-618.

Hu, P., Dhelim, S., Ning, H., and Qiu, T. (2017). Survey on fog computing: architecture, key technologies, applications and open issues. Journal of Network and Computer Applications, 98(April):27-42.

Iorga, M., Feldman, L., Barton, R., Martin, M., Goren, N., and Mahmoudi, C. (2018). The nist definition of fog computing. Technical report, National Institute of Standards and Technology.

Lewis, G. A. (2019). Edge computing: Use cases and challenges.

Mahmud, M. and Buyya, R. (2016). Fog Computing: A Taxonomy, Survey and Future Directions.

Mukherjee, M., Shu, L., and Wang, D. (2018). Survey of fog computing: Fundamental, network applications, and research challenges. IEEE Communications Surveys and Tutorials, 20(3):1826-1857.

Naha, R. K., Garg, S., Georgakopoulos, D., Jayaraman, P. P., Gao, L., Xiang, Y., and Ranjan, R. (2018). Fog computing: Survey of trends, architectures, requirements, and research directions. IEEE Access, 6:47980-48009.

Souza, A., Cacho, N., Noor, A., Jayaraman, P. P., Romanovsky, A., and Ranjan, R. (2018). Osmotic monitoring of microservices between the edge and cloud. In 2018 IEEE 20th International Conference on High Performance Computing and Communications; IEEE 16th International Conference on Smart City; IEEE 4th International Conference on Data Science and Systems (HPCC/SmartCity/DSS), pages 758-765. IEEE.

Syed, H. J., Gani, A., Ahmad, R. W., Khan, M. K., and Ahmed, A. I. A. (2017). Cloud monitoring: A review, taxonomy, and open research issues. Journal of Network and Computer Applications, 98:11-26.

Velasquez, K., Abreu, D. P., Goncalves, D., Bittencourt, L., Curado, M., Monteiro, E., and Madeira, E. (2017). Service orchestration in fog environments. In Proceedings 
- 2017 IEEE 5th International Conference on Future Internet of Things and Cloud, FiCloud 2017, volume 2017-Janua, pages 329-336.

Wen, Z., Yang, R., Garraghan, P., Lin, T., Xu, J., and Rovatsos, M. (2017). Fog orchestration for internet of things services. IEEE Internet Computing, 21(2):16-24. 